\title{
Introduction to the CPAIOR 2018 fast track issue
}

\author{
Willem-Jan van Hoeve ${ }^{1}$
}

Published online: 29 May 2018

(C) Springer Science+Business Media, LLC, part of Springer Nature 2018

The 15th International Conference on the Integration of Constraint Programming, Artificial Intelligence, and Operations Research (CPAIOR) was held in Delft, The Netherlands, from June 26 to June 29, 2018. It was co-located with the International Conference on Automated Planning and Scheduling (ICAPS).

The aim of the CPAIOR conference is to bring together interested researchers from Constraint Programming (CP), Artificial Intelligence (AI), and Operations Research (OR) to present new techniques or applications, and to provide an opportunity for researchers in one area to learn about techniques in the others. A main objective of this conference series is also to give these researchers the opportunity to show how the integration of techniques from different fields can lead to interesting results on large and complex problems.

All papers submitted to the conference were subject to a rigorous peer-review process. Accepted papers were either formally published in the conference proceedings [1], or invited to be published exclusively through a fast-track process in the Constraints journal. Only a small selection of outstanding papers was invited to the fast track. Before being accepted into Constraints, the resulting papers were subject to another round of reviewing to ensure the high standards for this journal. This issue collects the three papers that were accepted to the fast track:

The paper Deep Neural Networks and Mixed Integer Linear Optimization by Matteo Fischetti and Jason Jo studies the use of 0/1 mixed integer linear programming models for deep neural networks. The paper also analyzes a bound-tightening mechanism, and demonstrates the performance of the 0/1 MILP model on two applications in the context of feature visualization and adversarial machine learning.

This article belongs to the Topical Collection: Integration of Constraint Programming, Artificial Intelligence, and Operations Research

Guest Editor: Willem-Jan van Hoeve

Willem-Jan van Hoeve

vanhoeve@andrew.cmu.edu

1 Tepper School of Business, Carnegie Mellon University, 5000 Forbes Avenue, Pittsburgh, PA 15213, USA 
The paper Online Over Time Processing of Combinatorial Problems by Robinson Duque, Alejandro Arbelaez, and Juan F. Díaz proposes computational approaches to solving online machine scheduling problems, especially those that appear in the context of cloud computing. The paper uses a combination of machine learning, mixed integer programming, and instance interruption heuristics to maximize the number of requests that can be solved over time.

The paper Intruder Alert! Optimization Models for Solving the Mobile Robot GraphClear Problem by Michael Morin, Margarita P. Castro, Kyle E. C. Booth, Tony T. Tran, Chang Liu, and J. Christopher Beck develops optimization approaches to the graph-clear problem in which mobile robots are tasked with clearing a facility of intruders. The paper presents mixed-integer linear programming and constraint programming models, as well as new heuristic variants of the problem. The authors demonstrate that the optimization-based approaches outperform existing heuristics from the literature.

I thank the reviewers and the authors for their help and commitment which made it possible to have all papers published on time for the conference.

\section{References}

1. van Hoeve, W.-J. (Ed.) (2018). Proceedings of CPAIOR 2018, volume 10848 of Lecture Notes in Computer Science. Berlin: Springer. 